\title{
Technological Applications of the Grand Unified Theory
}

\author{
E. E. Escultura \\ Research Professor(Honorary), \\ GVP - Professor Lakshmikantham Institute for Advance Studies \\ GVP College of Engineering, JNT University Kakinada, Vishakhapatnam, AP India
}

\begin{abstract}
This paper presents the fundamentals of quantum gravity, one of the two pillars of the grand unified theory (GUT) and its technological applications. GUT is a physical theory, i.e., a mathematical space where the basic premises or axioms are laws of nature. Therefore, GUT inherits the rigor of mathematics, its language, as a deductive system. Proposed by Albert Einstein in the 1920s to unify the four forces of nature - gravity, electromagnetism and the weak and strong forces of quantum physics - it did not materialize until the discovery of the superstring, fundamental building block of matter (1997) as a necessary step to solve the 200-year old gravitational n-body problem. The solution yielded 9 natural laws of GUT beyond the four laws of thermodynamics, Newton's three laws of motion and his universal law of gravitation. True to its name, what started with the narrow objective of unifying the four forces of nature, GUT now unifies the natural sciences in the sense that every natural science field is an extension of GUT plus natural laws specific to that field. Moreover, every natural phenomenon (appearance of nature) is explained by natural laws. Most of all, GUT explains how nature works which makes it the basis for designing a class of technologies called GUT technologies. We focus on two of them:

(1) Technology for massive generation of electric power

(2) Magnetic charger for battery cars and vehicles.

Both technologies run on the free, clean, indestructible and inexhaustible dark matter that is abundant everywhere around us using magnets alone. Moreover, the paper offers improvement on the magnetic train which also runs on the clean, free and indestructible dark matter using magnets alone. Only China, Japan and South Korea own and operate magnetic trains.
\end{abstract}

Key Words. Magnetic Engine, Generalized Fractal, Primum, Quantum Gravity, Quark, Superstring.

\section{INTRODUCTION}

Originally proposed by Albert Einstein in the 1920s with the narrow objective of unifying the four forces of nature - gravitation, electromagnetism, the weak force and the strong forces - by a single set of equations that describe them; the project did not materialize during the next 85 years until the discovery of the superstring in 1997 [1]. The superstring is the fundamental building block of matter. The reason for the failure is two-fold: (1) the theory of relativity which Albert Einstein tried to use to pursue the project was critically flawed by the premise of empty space beyond the observable bodies in the cosmos and (2) inadequacy of the traditional method of computation and measurement. The existence of electromagnetic waves alone contradicts this premise because a wave needs a medium to propagate itself.

Science - the study of nature - has two components: the science itself, and its language mathematics. A field of science is defined by its subject matter. The traditional method of mathematics has been computation and measurement. It is inadequate for complex situation that often arises in mathematics. This is the reason traditional mathematics failed to solve or 
resolve long standing problems such as the 360-year-old Fermat's conjecture (popularly known as Fermat's last theorem [2]) and the 250-year-old Goldbach's conjecture [3]. That is why the author had to apply a new methodology for mathematics that he introduced in [4] called qualitative mathematics to make a critique-rectification of the underlying fields of FLT that started in 1992 and culminated in its resolution in 1998 by way of a counterexample that proved FLT false [5].

Qualitative mathematics, the mathematical model of rational thought [6] and the complement of computation and measurement, was applied to physics in 1996 to devise a new methodology - qualitative mathematics and modeling - that yielded the solution of the gravitational n-body problem [1]. Qualitative mathematics involves the following activity:

Making conclusions, visualizing, abstracting, thought experimenting, learning, creating concepts for building mathematical a space, intuition, imagination, trial and error to sift out what is appropriate, negating what is known to gain insights into the unknown, altering premises to draw out new conclusions, thinking backwards, finding basic premises for a mathematical space and devising techniques that yield results.

Qualitative mathematics releases thought from constraint in the pursuit of knowledge. However, since it also raises the chance of error, flaw and ambiguity creeping in, it must provide safeguards against this possibility. It does by making every field of mathematics a mathematical space built on consistent basic premises or axioms. A mathematical space consists of concepts (e.g., symbols, words, figures), operations and relations where every concept, operation and relation is defined by the axioms. It is a deductive system, i.e., every conclusion (e.g., theorem) is supported by and follows from the axioms. Reference [7] enumerates the known sources of ambiguity, some of which inherent in certain concepts (e.g., infinity), and how to contain them.

In science the traditional method of quantitative modeling (formerly called mathematical modeling) describes natural phenomena (appearances of nature) mathematically and uses reasoning by analogy which is unreliable. This method is inadequate and left long standing problems of physics unsolved. The author applied qualitative mathematics and modeling to physics for the first time to solve the n-body problem and launch, at the same time, the development of the grand unified (GUT). To avoid error, flaw and ambiguity we build every field of science as a physical theory, i.e., a mathematical space where the axioms are laws of nature. This way, science inherits the precision of mathematics as a deductive system.

\section{OVERVIEW OF THE GRAND UNIFIED THEORY}

We provide a sweeping summary of quantum gravity (details and explanation in [8]), one of the two pillars of GUT, the other macro gravity concerned with cosmological bodies.

\section{The Superstring}

We first introduce partial definition of physical concepts until the discovery of appropriate natural laws that define them are found. Energy is motion of matter. Therefore, matter and energy are never separate and neither pure matter nor pure energy exists. Thus, there is no massless piece of matter and the photon has mass. Once the superstring is discovered, matter is known and everything else is. The semi- and non-agitated superstrings comprise dark matter, one of the two fundamental states of matter, the other ordinary or visible matter. Dark matter is not directly observable because its constituent superstring has wavelength less than that of visible light (less than $10^{-16}$ meters). Flux is motion of matter with direction at each point. Wave is suitably synchronized sequence of resolution of contending forces in the medium that results in suitably synchronized vibration of the medium. In the case of water 
wave the water molecules are the medium and the contending forces are gravity and pressure. Chaos is mixture of order none of which is identifiable. For example, chaos occurs at the onset of a hurricane. When a huge solid el niño forms on the ocean surface (a region of warm surface due to under-ocean volcanic eruptions) the lower atmosphere above it heats up causing low pressure (depression). Heat raises kinetic energy and motion, pushes the molecules apart, lowers density and reduces the pressure. The atmospheric depression sucks the air molecules around it by the trillions. At this phase in the evolution of this phenomenon it is impossible to identify the path of a single molecule among the trillions due to their collision (chaos) but every molecule is subject to natural laws (order). This is the first formal definition of chaos. Since chaos is energy dissipating due to collision, Energy Conservation pushes this phase towards a global order, a coherent vortex in the atmosphere called hurricane which is turbulence. Electromagnetic wave, one of the two basic cosmic waves, is generated by the natural vibration of atomic nucleus and propagated across dark matter. The natural vibration of the atomic nucleus is due to the impact of electromagnetic waves coming from all directions in the cosmos. Thus, every piece of matter has natural vibration.

\section{The Laws of Nature}

We include Newton's three laws of motion [9] and the four laws of thermodynamics [10] among the laws of nature but we introduce more natural laws that we need. We first state the fundamental natural law, a modification and extension of the first law of thermodynamics [11] to dark matter.

Energy Conservation Law. In any physical system and its interaction, the sum of kinetic (visible) and latent (dark) energy is constant, gain of energy is maximal and loss of energy is minimal.

This law is fundamental because all other natural laws and principles are expressions of it. We express this as a natural law.

Energy Conservation Equivalence. Energy conservation has many expressions or forms: order, symmetry, economy, least action, optimality, efficiency, stability, self-similarity (nested fractal), coherence, resonance, quantization, synchronization, smoothness, uniformity, motionsymmetry balance, non-redundancy, non-extravagance, evolution to infinitesimal configuration, helical and related configuration such as circular, spiral and sinusoidal and, in biology, genetic encoding of brain wave vibration characteristics, reproduction and order in diversity and complexity of functions and configuration that provides optimal capability.

Each component of this law is called physical or mathematical principle. Being circular, sinusoidal and symmetrical are universal (natural) configurations of matter according to the Energy Conservation Equivalence law. That is why the profile of a wave is sinusoidal, not triangular or trapezoidal. Consider a piece of rock thrown into the water. It pushes molecules of water down with it. However, pressure pushes them upwards and above the water surface due to momentum (product of mass and speed). Gravity pulls them down below the surface due to momentum. Pressure pushes them up again, etc., causing oscillation about the water surface. Water viscosity induces formation of advancing circular molecular oscillation (vibration) around the point of impact causing a sequence of concentric circular waves propagated away from the point of impact. Water viscosity has a damping effect on the molecular vibration, dissipates the energy imparted by the impact of the falling rock and soon the waves vanish. Similarly, electromagnetic wave is suitably synchronized vibration of the medium. This definition rules out wave in empty space. The characteristic of nuclear vibration is determined by the nuclear structure in accordance with this natural law: 
Internal-External Factor Dichotomy Law. The interaction, dynamics and physical characteristics of a physical system are shaped by internal and external factors; in general the internal is principal over the external and the latter works through the former.

Heat is kinetic or visible energy due to vibration of atoms and molecules of a body. Its propagation through heat conduction is in accordance with the Resonance Law [11] that says, Resonance Law. Maximum resonance between waves, oscillation or vibration occurs when they have exactly the same characteristics with wavelength or frequency as the principal factor. The degree of resonance declines with the difference between wave characteristics and orders of magnitude of their wavelengths. However, at suitably high order of magnitude of wavelength the infinitesimal effect of resonance by orders of magnitude nearby rises to significance.

The next natural law is crucial for the study of turbulence.

Flux-Low-Pressure Complementarity Law. Low pressure sucks matter around it and the initial chaotic rush of matter towards a region of low pressure stabilizes into local or global coherent flux called turbulence; conversely, coherent flux induces low pressure around it.

This natural law was inspired by a simple high school experiment many years ago.

One error of traditional physics that delayed the resolution of the ancient quest for the superstring is the failure to identify the requirement for the fundamental building block of matter which is indestructibility. Otherwise, our universe would have vanished long ago which did not for it has existed for 8 billion years (corrected value) [12].

It is a fact that matter appears steadily and rapidly, especially, in certain regions of the cosmos called star nurseries [13,14]. By Energy Conservation, it must have come from somewhere and that is what we have called dark matter consisting of non-agitated and semi-agitated superstrings. The only force that resonates with the superstring is electromagnetic wave which is fractal being generated by the fractal atomic nucleus [15]. Hit by suitable electromagnetic wave a non-agitated superstring is (a) thrown by its impact; bounces with others and comes to rest in dark matter when the imparted energy dissipates or (b) gets close to its path and sucked by it, by Flux-Low-Pressure Complementarity, and forms a loop the original superstring becoming its toroidal flux. By Energy Conservation and Energy Conservation Equivalence, its path shrinks and evolves to energy-conserving form: closed circular helical spiral loop with a superstring traveling through its cycles at $7\left(10^{22}\right) \mathrm{cm} / \mathrm{sec}$ [16] called toroidal flux; this value is a constant of nature for toroidal and induced vortex fluxes. Energy Conservation Equivalence requires this structure be repeated in every toroidal flux from which follows the fractal sequence structure [17] of the superstring which makes it indestructible. We identify the semiagitated first term with the superstring itself because it is the term involved in its interactions. (c) Hit by suitable electromagnetic wave the first term of the superstring expands and becomes semi-agitated, by Energy Conservation. In both cases (a) and (b) the superstring is a nested generalized physical fractal sequence of superstrings [17]. Its latent energy is super huge due to its fractal structure. The non-agitated superstring is mathematically modeled by the $d$ sequence of the dark number $\mathrm{d}^{*}[18]$.

We summarize our findings as a natural law.

Existence of Fundamental Building Block of Matter and its Generalized Nested Fractal Structure. The fundamental building block of matter is the superstring, a non-agitated superstring traveling at $7\left(10^{22}\right) \mathrm{cm} / \mathrm{sec}$ through its helical circular loop and nested fractal 
sequence of superstrings with itself as first term, each superstring except the first is contained in and similar to the preceding term in structure, behavior and properties; each superstring except the first is contained in and similar to the preceding term in structure, behavior and properties; each superstring except the first is contained in and similar to the preceding term in structure, behavior and properties, etc., ...

The figure described is called nested generalized physical fractal sequence [17], the structure that makes the superstring indestructible (Figure 1).

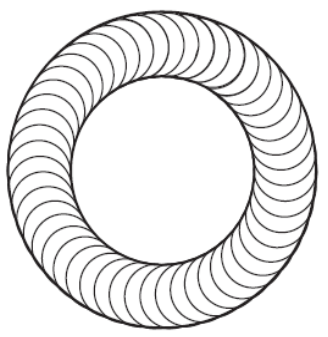

Figure 1: A semi-agitated superstring; its dark toroidal flux travels through its cycles at $7\left(10^{22}\right)$ $\mathrm{cm} / \mathrm{sec}$. (Figure taken from [19])

The superstring is non-agitated if its cycle length (CL) is less than $10^{-16}$ meters, semi-agitated if $10^{-16}<\mathrm{CL}<10^{-14}$ meters and agitated if its CL $>10^{-14}$ meters. Dark matter consists of nonagitated superstrings.

\section{THE PRIMUM}

When suitable electromagnetic wave hits a semi-agitated superstring a pair of mutually exclusive events occurs: (d) the first term of its fractal superstring bulges to retain the toroidal flux speed despite the energy imparted by the electromagnetic wave, by Energy Conservation, turning it into an agitated superstring called primum, unit of visible matter, its toroidal flux non-agitated, or (e) the first term breaks, its toroidal flux remaining non-agitated and dark. The primum has dark superstring component that attaches it to dark matter through resonance. The basic prima are the electron, charge -1 ; +quark, charge $+2 / 3$; and -quark, charge $-1 / 3$ [20]. They are basic because they comprise every atom of our universe.

The next law articulates our findings.

Dark-to-Visible-Matter Conversion. When suitable electromagnetic wave hits a semi-agitated superstring one of these occurs: (i) the outer superstring breaks, its toroidal flux remaining nonagitated superstring; (ii) a segment bulges into a primum, an agitated superstring and a unit of visible matter.

When a suitable electromagnetic wave hits a superstring the first term of the sequence breaks, by Resonance, leaving the rest intact and nested fractal sequence of superstrings, i.e., a superstring. Thus, this structure insures its indestructibility. It is neither created nor destroyed; it only goes through an endless cycle. It follows that the Universe of dark matter has no beginning and no end (timeless). By Flux-Low-Pressure Complementarity, it has no boundary, i.e., unbounded and infinite, and our universe is a finite local bubble in it among other universes.

The next natural law is central to primal interaction. We first state its broad form. 
Flux Compatibility*. Two fluxes of the same direction attract but two fluxes of opposite directions repel each other.

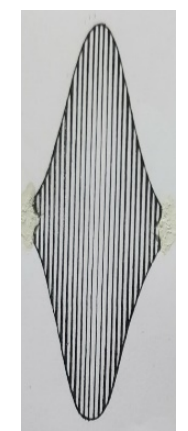

Figure 2: A simple primum, bulged segment of a semi-agitated superstring. Its toroidal flux pulls and spins the non-agitated superstrings around it (by Resonance) into its magnetic flux. Its polarity conforms to the right-hand-rule of electromagnetism: when the index finger points in the direction of its toroidal flux, the thumb points to the north pole. (Figure taken from [19])

We state a special form of the Flux Compatibility* law that applies directly to vortex fluxes of superstrings.

Flux Compatibility. Two prima of opposite toroidal flux spins attract at their equators but repel at their poles; otherwise, they repel at their equators but attract at their poles. Two prima of same toroidal flux spin connect equatorially only through a primum of opposite toroidal flux spin between them called connector.

A physical system is observable through the medium of light if its size is comparable with the wavelength of the latter; this follows from the Resonance Law. Thus, a semi- or non-agitated superstring is not observable since its cycle length is less than the finest wavelength of visible light in order of magnitude which is about $10^{-14}$ meters.

\section{The Induced Vortex Flux of the Primum}

What really is a primum? When the toroidal flux along its cycles is hit by cosmic waves coming from all directions it is thrown into erratic motion due to collision with other superstrings turning it into a spike with the centroid traveling through the cycles at $7\left(10^{22}\right) \mathrm{cm} / \mathrm{sec}$. It pulls the superstrings around the primum into its induced vortex flux (simply referred to as vortex flux), by Flux-Low-Pressure Complementarity, with its axis coinciding with the axis of the primum inside the cylindrical eye making it a magnet, its polarity in accordance with the righthand rule of electromagnetism. The primum in Figure 2 is actually wrapped by the spinning vortex flux which is not shown being dark. The vortex flux is counterclockwise for a positive primum, by convention, negative otherwise. By Newton's action-reaction law [9] a free positive primum has the opposite spin (spin of quantum physics and a measure of angular momentum, e.g., the electron has spin $+1 / 2$ [21]; distinguished from vortex flux spin). The plane passing through the apex of the primum's profile normal to its axis is the equatorial plane, its intersection with the primum the equator.

The energy of the vortex flux of a primum is measured as charge; the energy of the electron is $\left(1.6\left(10^{-19}\right)\right.$ coulombs $)$. Charge is a property of the vortex flux of the primum that follows from Flux-Low-Pressure Complementarity. The energy of the induced vortex flux of a primum is measured as charge its unit the electron's charge: -1 . Seismic waves generated by the micro component of turbulence at its interfaces and at the spinning cores of a cosmological vortex $[22,23]$ convert dark to visible matter in and around it as cosmic dust. They congeal into stars. 
The primum's toroidal and vortex fluxes as well as its natural vibration endow dark matter huge latent energy partially convertible to kinetic energy. The latent energy density of dark matter is $10^{26}$ joules/cubic ft. according to de Broglie [24], 8(108) volts/cm says Seike Jr [24] and the equivalent of $18 \mathrm{~kg} / \mathrm{cu}$ meter according to Gerlovin [20] using relativistic conversion.

\section{PRIMAL INTERACTION}

The proton consists of two +quarks joined by -quark equatorially, by Flux Compatibility (Figure 3). By Energy Conservation, their axis are coplanar; the proton's charge: $2 / 3-1 / 3+$ $1 / 3=+1$. Thus, the proton has net coherent counterclockwise vortex flux around it. Since a simple primum is charged, the neutral neutrino must be a coupled pair of simple prima of numerically equal but opposite charges, say, $+q$ and $-q$, so that its charge is $+q+-q=0$, neutral.
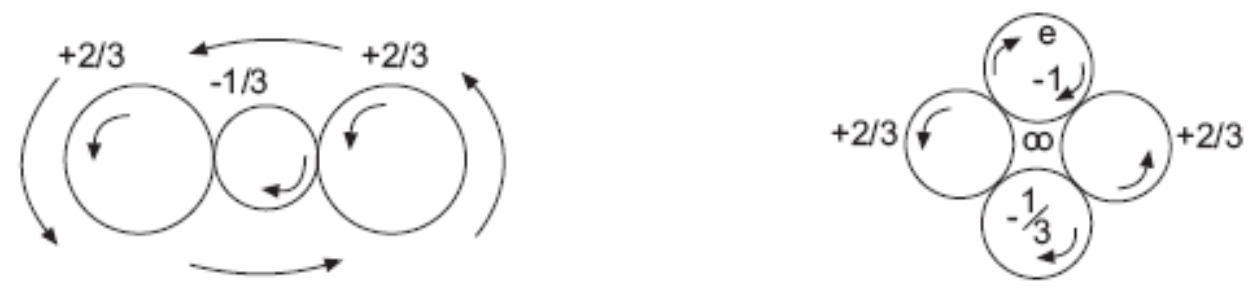

Figure 3.The proton is a cluster of two +quarks joined by a -quark at their vortex flux's rims by Flux Compatibility, left. The neutron (right) consists of a proton, electron and neutrino (represented by the horizontal figure 8 at the center of the right figure). Thus, the two +quarks of the proton are joined separately by the electron and -quark (by Flux Compatibility). The coherent flux at the center of the neutron (same direction of spin at interfaces) creates suction, by Flux-Low Pressure Complementarity, that pulls the neutrino in. (Figure taken from [19])

By Flux Compatibility the electron can attach itself to a positive quark of the proton but Energy Conservation and the optimality principle of the Energy Conservation Equivalence law require that it attaches to both + quarks beside the negative quark as the most stable position but pushes the -quark a bit by Flux Compatibility so that their centers viewed from the north pole form the vertices of a quadrilateral. In its interior are the coherent vortex fluxes of the +quarks, -quark and electron that make it a region of low pressure or depression. By Flux-Low-Pressure Complementarity its interior sucks a neutral primum around it since charged primum is repelled by the -quark or +quark. Therefore, only suitably light neutral primum fits in and that is the neutrino. Thus, we have just reconstructed the neutron consisting of a proton, electron and neutrino. Its charge: $+2 / 3-1 / 3+2 / 3-1+0=0$, i.e., neutral, and there is no net coherent vortex flux around it. The vortex flux of a charged coupled primum is extended along the equatorial plane due to greater centrifugal force there [16].

\section{THE ATOM, MOLECULE, HEAVY ISOTOPE}

The protons are the first to form the nucleus of the atom (Figure 4). When there is only one proton it coincides with the eye of its vortex flux. If there are more their vortex fluxes add up to form the vortex flux around it. The protons are joined pairwise by -quarks. Clearly, the nucleus is fractal; therefore, the electromagnetic waves generated by its natural vibration is also fractal and endowed with huge energy. As positive coupled primum the nucleus is a magnet of positive polarity with the vortex flux around it providing the magnetic field. Viewed from the north pole the vortex flux of a free atom spins counterclockwise (right hand rule of electromagnetism). The electrons being negatively charged are attracted to the vortex flux away from the eye but being light, they are swept into orbit by the vortex flux. By centrifugal force, the most energetic orbital electrons are those closest to the equatorial plane; they form the outermost subshells [16]. The least energetic ones cluster near the poles and form the 
lowest orbital shells. A stable atom has orbital electrons equal to the number of protons in the nucleus. Otherwise, it is a positive or negative ion and reacts with other prima. Moreover, the eye sucks non-agitated superstrings that accumulate steadily in the nucleus as mini black hole [16], the principal source of nuclear energy in nuclear fission. In fact, every charged primum, simple or coupled, sucks and accumulates a mini black hole in the eye of every charge component. This was confirmed at CERN [25] by the great burst of energy attributed to the Higgs boson released when two protons collided at great speed. The neutron, being neutral, is the only primum sucked by the nuclear eye to form heavy isotope.

The usual molecular formation has one valence electron and a -quark, from each of the two atoms at their outer subshells that serve as double connector [16] so that the molecule has two electron connectors. Particularly strong bonding like the carbon molecule involves two subshells with double connectors.

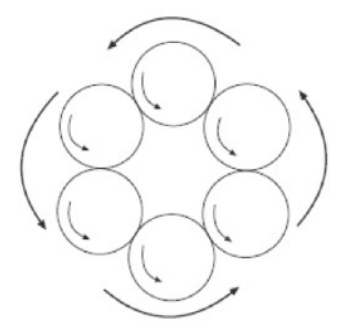

Fig. 4: Light nucleus viewed from the north-pole. The neutron is not indicated as it has no vortex flux being neutral. (Figure taken from [19])

Primal interaction is governed by Flux Compatibility and Flux-Low-Pressure Complementarity.

Since the masses of the neutron, proton and electron are known [26] and the composition of the neutron is known, the mass of the neutrino is computed as follows:

(1) neutron: $1.674\left(10^{-27}\right) \mathrm{kg}$; proton: $1.672\left(10^{-27}\right) \mathrm{kg}$; electron: $9.611\left(10^{-31}\right) \mathrm{kg}$.

Converting to atomic mass unit (amu) their masses are:

(2) neutron: $1.0087 \mathrm{amu}$; proton: $1.0073 \mathrm{amu}$; electron: 5.486(10-8) amu.

The mass of the neutrino is: $\eta=8.5\left(10^{-8}\right)$ amu or 1.55 times the electron's mass (that of the electron is $1 / 1840^{\text {th }}$ that of the proton's). Ironically, the mass of the neutrino is still a subject of hot pursuit, $[27,28]$.

A primum in flight rides and is lodged between tangent arcs of two electromagnetic waves at half-phase difference (Figure 5), its envelope. In cylindrical coordinates the primum has the equation $\left.\mathrm{x}=\mathrm{t}, \mathrm{y}(\mathrm{t})=\beta\left(\sin ^{\mathrm{n}} \pi \mathrm{t}\right)\left(\cos ^{\mathrm{m}} \mathrm{k} \pi \mathrm{t}\right)\right), \theta=\mathrm{n} \pi \mathrm{t}, \mathrm{t} \in[-1 / \mathrm{k}, 1 / \mathrm{k}], \mathrm{n}, \mathrm{m}, \mathrm{k}$, integers, $\mathrm{n}>\mathrm{k}, \mathrm{m}$ even [30]. Its cycle energy is the Planck's constant $h=6.64 \times 10^{-34}$ joules. Scooped up and carried by cosmic wave, its cycles flatten to the rapid oscillation, $\mathrm{z}=0, \mathrm{x}=\mathrm{t}, \mathrm{y}(\mathrm{t})=\beta\left(\sin ^{\mathrm{n}} \pi \mathrm{t}\right)\left(\cos ^{\mathrm{m}} \mathrm{k} \pi \mathrm{t}\right)$ due to dark viscosity. Thus, a primum in flight is a fine sinusoidal arc embedded between a pair of electromagnetic waves (Figure 5). It becomes the photon, $\mathrm{z}=0, \mathrm{y}(\mathrm{t})=\beta\left(\sin ^{\mathrm{n}} \pi \mathrm{t}\right)\left(\cos ^{\mathrm{m}} \mathrm{k} \pi \mathrm{t}\right)$, when it breaks off from its loop; the energy of one full cycle of the primum or one full arc of photon (its projection on the plane) is $\mathrm{h}$ and its toroidal flux speed along the $\operatorname{arc}$ is $7\left(10^{22}\right) \mathrm{cm} / \mathrm{sec}$. 

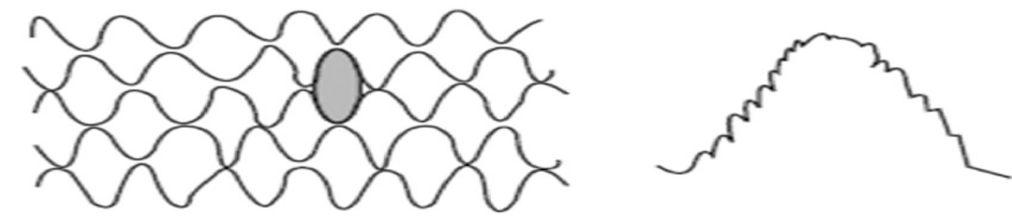

Fig. 5. A primum or photon lodged between two arcs of parallel electromagnetic waves, left. An arc of electromagnetic wave, right, fractal being generated by the natural vibration of the fractal nucleus. The fractal structure of electromagnetic wave endows it with huge energy that it can penetrate concrete wall, e.g., radio waves.

Since the photon has energy, it has mass converted from the mass of the primum it comes from; its speed equals the speed of its electromagnetic wave carrier which is $3\left(10^{10}\right) \mathrm{cm} / \mathrm{sec}$ after adjusting for the effect of dark viscosity. Not being a loop, the photon collapses when it leaves the carrier wave envelope its toroidal flux remaining dark. This is the reason it has no rest mass. This happens when the photon is blocked by opaque substance; its toroidal flux breaks away from its carrier (which goes through) and remains dark and non-agitated. In large quantity the combined kinetic energy heats up the opaque medium. In translucent medium some of the photons go through and remain in their carriers. When the photon is knocked off by another cosmic wave or reflected by a mirror it switches to another electromagnetic wave carrier in the direction of transit.

\section{QUANTUM PHENOMENA}

We present some of the unexplained natural phenomena in traditional physics.

\section{Wave-particle duality}

The wave-particle duality of quantum physics applies to any primum or photon that is embedded between adjacent parallel electromagnetic waves their ends half-phase apart, e.g., electron or photon (Figure 5). That the electron is a particle is well established. That it is a wave is supposedly confirmed by shooting a beam of electrons through slits in a thin plate where they become waves emanating from the slits on the other side of the plate [30], an electron in the beam supposedly becoming a wave.

The notion that a particle is both wave and particle is a contradiction. A particle is an autonomous physical system independent of the medium while a wave is suitably synchronized vibration of the medium with a sinusoidal profile expanding radially from its source (analogous to the "motion" of neon lights due to synchronized sequence of switching); each vibrating element remaining in place. That medium is dark matter which is unknown in traditional physics including quantum physics. Therefore, this contradiction cannot be resolved there. With dark matter as medium the contradiction is removed. The wave characteristic is provided by the carrier electromagnetic wave and the particle characteristic by the primum it carries.

\section{Matter-anti-matter interaction}

Two simple prima are anti-matter to each other if they are mirror images of each other with respect to a plane between them normal to their common equatorial plane. For example, the electron and positron are anti-matter of each other. Their toroidal flux spins are symmetric and have opposite directions. Therefore, they attract each other. When they get close, the momentum of their attraction forces their cycles to overlap and their fluxes to repel (since their overlapping cycles have opposite toroidal flux spins) leading to explosion, by Flux Compatibility, that throws them as photons into opposite directions parallel to their respective axis. The logic of GUT says that every simple primum has anti-matter. However, primal polarity and the Earth's gravitational flux separate matter from its anti-matter. Free negative anti- 
matter, e.g., the electron and -quark, remains on the ground while light free positive antimatter remain in the upper atmosphere from the mesosphere to the fringe of the Earth's gravitational flux.

A simple primum has anti-matter. Does a coupled primum have anti-matter? Yes when it has suitable symmetry. For example, since the two +quark and the -quark of the proton are linearly arranged, a coupled primum consisting of two -quarks joined by a +quark is its antimatter. Then they are attractive and when close, the momentum of their approach forces their cycles to overlap and mutually throw each other in opposite direction. Such occurrence is minimized by the Earth's gravitational flux which separates them, the negative prima on the ground and the positive prima at the fringe of the Earth's gravitational flux. Neutral prima, of course, have no anti-matter but their charged components have. However, a simple primum even if coupled with other prima may be annihilated by its anti-matter. This applies to charged components of the neutron. In fact, individual simple primal component of a coupled primum have anti-matter that can mutually destroy each other.

\section{The Higgs boson}

The recent experiment at CERN released powerful burst of energy upon head-on collision of two protons at great speed, each close to the speed of light, and this was attributed to the Higgs boson within the proton. To be precise, what the experiment revealed was the destruction of that so-called Higgs boson. Therefore, the Higgs boson cannot be the fundamental building block of matter. It is actually one of the three mini black holes in the eyes of the +quarks and -quark that comprise the proton [16] and the result of the experiment can be examined to check if one, two, three, four, five or six Higgs bosons were exploded by the collision since the two +quarks and the -quark of each proton have their respective mini black holes. The experiment confirms the existence of a mini black hole in a simple primum. When suitably agitated it converts to huge burst of kinetic energy, e.g., photons. The experiment is also an independent verification of the mini black hole as source of tremendous energy released by nuclear explosion.

\section{Primal Polarity}

We look at the primum as it pops out of dark matter. Since the dark superstring has infinitesimal induced vortex flux, it has no polarity and does not interact with anything except cosmic waves and it is oriented randomly. When a dark superstring is agitated by suitable cosmic wave and pops out of dark matter as free positive primum and its equatorial plane is oblique to the direction of the gravitational flux it rotates suitably due to the coriolis effect [22.31], its north pole pointing North and equatorial plane parallel to the Earth's equatorial plane making its vortex flux a counterclockwise eddy in the gravitational flux (which spins from West to East), its optimal energy-conserving alignment. By Flux Compatibility, free positive prima are pushed up so that there is abundance of free positive prima in the upper atmosphere. Free neutral prima are oriented randomly but free positive ions are counterclockwise eddies in the Earth's gravitational flux; they are pushed upwards by Flux Compatibility. However, the protons being heavy remain in the lower atmosphere. The free electrons as clockwise eddies in the Earth's gravitational flux whose equatorial plane is parallel to the Earth's equatorial plane are pushed downwards, by Flux Compatibility. Thus, there is abundance of free electrons on the ground. Other free negative prima including the -quarks should be abundant on the ground also but we do not know where they are and no study has been done on them. When the voltage between the positive ions in the lower atmosphere and the electrons on the ground reaches critical level they rush towards each other, collide and explode as lightning. Lightning, being at the interface of turbulence 
generates seismic waves that convert dark matter to earthlights in the mesosphere called sprites, elves, blue jets and gamma rays [32]

\section{Brittle and malleable materials}

Brittle material, e.g., ceramics, has no free electrons; therefore, it is good electrical insulator and does not vibrate and transfer heat. That is why it is heat insulator. Malleable material like metal has free electrons; therefore, it is good electrical conductor. When bent valence electrons are expelled but when released free electrons replace them, the material restored to its original form the essence of elasticity. Therefore, malleable material vibrates as synchronized sequence of distortion and "restortion". When heated at a point malleable material vibrates there since heat is vibration of molecules and, through resonance, vibration spreads outward along concentric circles (if its elasticity is homogenous) and heat is similarly conducted outwards. Therefore, malleable material is good heat conductor.

When metal vibrate beyond a critical point and the rate of expulsion of valence electrons is greater than the rate of replacement it may soften or even melt. Valence electrons may also be expelled by the impact of seismic waves. The softening of metal attachments of building foundations and concrete reinforcement due to high intensity seismic waves during earthquake knocks off buildings and cracks or pulverizes concrete [22]. It is not due to the usual gentle rocking action during earthquake that causes destruction but the impact of seismic waves. There is technological possibility here: making suitable alloy resistant to the softening impact of seismic waves and composite resistant to cracking and pulverization by them. It offers the possibility for constructing earthquake-proof structures.

\section{Metal fatigue}

An American Airlines plane's pylon broke and the engine fell off just after take-off from O'Hare International Airport in Chicago in the 70s causing the plane to crash and kill all 392 passengers aboard. Bridges suddenly collapse and metallic casing of a train's wheel breaks off, gets stuck dangling under the train and caught at rail junction causing terrible derailment accident (this happened in Germany three decades ago).

Metal is not perfectly elastic; when subjected to repetitive distortion like vibration, the net loss of valence elections accumulates over time, reaches a critical point and the metal snaps. This explains the cause of the above accidents involving repetitive motion. This is illustrated by a metallic paper clip that snaps when bent back and forth. Infinitesimal but progressive deterioration of vibrating metal is not detectable. Therefore, test of material should be made at appropriate conditions for its use to determine when metal fatigue sets in to avoid accident.

The greater elasticity of carbon relative to other gases due to stronger bonding raises its normal vibration frequency and absorption of excess heat in the atmosphere coming from the Sun contributes significantly to the Greenhouse effect [33].

\section{ELECTROMAGNETISM}

Every minute of one's life depends on electricity and magnetism and every engineer knows their relationship: magnet produces electricity and electricity produces magnet. Today, we lift technology into a new domain and resolve the burning issue of the moment: climate change.

\section{Magnet}

Recall that the atom is a magnet with a vortex flux of superstrings powered by the protons in the nucleus. Its polarity is in accordance with the right-hand rule of electromagnetism. Denote by $\mathrm{N}$ and $\mathrm{S}$ its north and south poles. Using the right-hand rule of electromagnetism the spin of 
its vortex flux is counterclockwise looking at it from the north pole $\mathrm{N}$. In ferromagnetic material, e.g., iron, most atoms can be aligned and joined N-to-S or S-to-N to form a string. Then the string can be joined equatorially with other strings through their component atoms by -quarks to form a bundle, the maximum number of strings depending on the material, by quantization. In ordinary magnet each bundle determines a line of force whose induced vortex fluxes are subject to the righthand rule. Both $\mathrm{N}$ and $\mathrm{S}$ poles of a bar magnet have little $\mathrm{N}$ and $\mathrm{S}$ poles corresponding to the poles of the bundles. They show when iron filings are dropped sparingly on a piece of thin paper placed over the magnet. The coherent vortex flux of the bundles creates a magnetic field. In a magnet the bundles induce their extensions beyond the N-pole to form extensions of their strings (by Flux Compatibility) that go around to join their respective south poles. The bundles form the lines of force in a magnet. They can be mapped by dropping iron filings sparingly on a piece of thin paper placed over the magnet.

\section{Electric Current}

When a live conductor (malleable material like metal) cuts across the magnetic lines of force, vortex flux of superstrings flow through it at speed of $7\left(10^{22}\right) \mathrm{cm} / \mathrm{sec}$. In a malleable material there are free electrons because it has low ionization energy. This means that orbital electrons are knocked off their orbits by electromagnetic waves. They ride on the flux of superstrings. When they collide with atoms of a resistor, e.g., the filament of an electric bulb, they produce heat or light. When the temperature of the conductor is reduced to $110^{\circ} \mathrm{K}$ or less, the ionization energy rises so that free electrons vanish, i.e., there is electric current (flux of superstrings) without resistance because there are no electrons to collide with he atoms of the resistor. This phenomenon is called superconductivity [34].

In a conventional power plant a live conductor (closed circuit) is coiled around an armature and rotated so that the coils cut magnetic lines of and generate electricity. Alternating current is produced. Currently, fossil, nuclear and geothermal fuel run the motor that that rotates the armature. In a conventional car battery, the electrolyte ionizes the cathode (positive terminal) and the releases electrons move to the anode (negative terminal). The terminal marked negative is the source of electrons that when connected to an external circuit will flow and deliver energy to an external device. When a battery is connected to an external circuit, electrolytes are able to move as ions within, allowing the chemical reactions to be completed at the separate terminals and deliver energy to the external circuit.

\section{Making a magnet}

To make a bar magnet, one wraps a coil around it connected to an alternating current. Then another coil is wrapped over the first and connected to direct current. When power is turned on for both the first coil shakes the atoms, the second coil aligns and joins them polarly to form strings; -quarks join strings to form bundles and the iron bar becomes a magnet. A strong magnet can be made by raising the voltage and duration of the process. The alternating current is turned off first when the charging is done. 


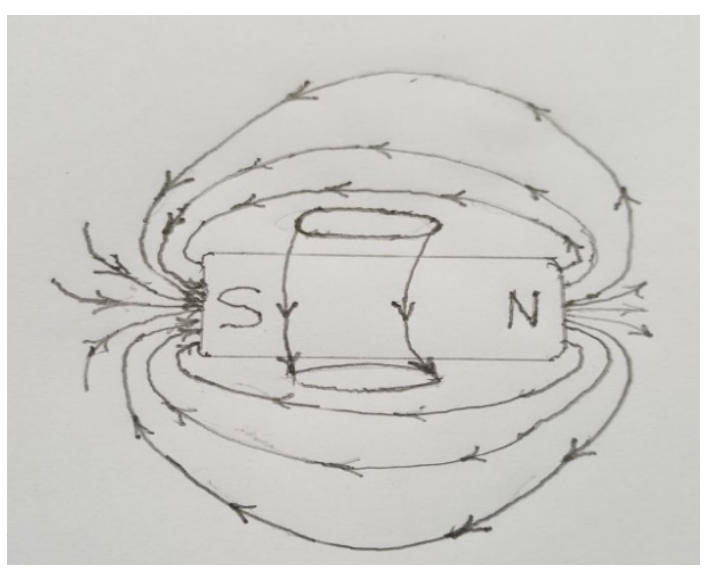

Figure 6. A bar magnet and its magnetic lines of force. It has a cylindrical eye in its magnetic flux around it. But the main eye is where the magnet is.

Magnets of the same polarity cannot be placed side by due to repulsion between them according to

Coulomb's law:

(3) $\mathrm{F}=\mu \mathrm{q}_{\mathrm{m} 1} \mathrm{q}_{\mathrm{m} 2} / 4 \pi \mathrm{r}^{2}$,

where

$F$ is force (SI unit: newton);

$q_{m 1}$ and $q_{m 2}$ are the magnitudes of magnetic poles (SI unit: ampere-meter);

$\mu$ is the permeability of the intervening medium (SI unit: newton per ampere squared);

$r$ is the separation (SI unit: meter).

Therefore, to put them side by side requires magnetic insulators between adjacent magnets.

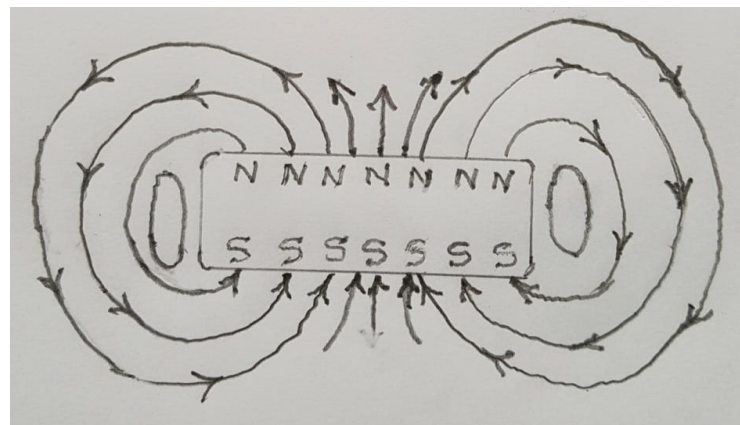

Figure 7. Fat bar magnet. A bar magnet has little north poles of their bundles at its north pole. Each bundle extends beyond the north pole and if strong enough goes around as shown by the arrows to connect with its south pole. For the magnetic train (maglev) elliptical magnets are used.

Magnets in general have many industrial uses and fat bar magnets are particularly useful to hold and guide high-speed train like the maglev.

\section{Magnet Insulator}

A magnet insulator consists of thin narrow strips of ferromagnetic material scattered randomly (with respect to direction or orientation) but evenly on a flat surface heater. It is pressed by a similar flat surface heated just enough to weld the strips together. 


\section{THE MAGNETIC TRAIN OR MAGLEV (MAGNETIC LEVITATION)}

The author is not privy to the construction of the magnetic train or maglev (magnetic levitation). Therefore, this is only a recommendation for making the safest, most efficient and comfortable maglev based on GUT.

The ultimate in technology is one that uses the clean, free, inexhaustible and indestructible dark matter that comprises $95 \%$ of the cosmos and is abundant everywhere around us. In the case of technology for treatment of diseases the ultimate one has further requirement that it does not harm normal cells, i.e., it has no side effect. The pace-setter in ultimate technology is the maglev (Fig. 8). Here is how to construct it.

An array of parallel elliptical bar magnets is placed at the center of the track (length of magnets to fit between the track lids, see Figure 10), normal to and on the track, and line the full stretch of the track. The north pole of each magnet points to the left side of the track. Under the platform of each car are two elliptical bar magnets, 3/4 the length of the car apart at the middle, same length as the track magnets and directly above them, with the same polarity as the ones on the track. Since the track and platform magnets have the same polarity they are repulsive, i.e., the fluxes of the platform magnets and those of the track magnets push against each other, by Flux Compatibility. In effect, when the lateral axes are parallel to those of the track magnets, the train floats on the magnetic fluxes of the track magnets so that there is no friction when the train is pulled by the train's engine.

The maglev's "engine" is an elliptical bar magnet with the same polarity as the track magnets its planar axis horizontal when the train is at rest (Fig. 9).

When the magnetic engine is tilted downward the horizontal component of the repulsive force of the track magnets pulls the train forward. Since the maglev floats on magnetic flux there is no friction and its potential maximum speed is $1.4\left(10^{23}\right) \mathrm{cm} / \mathrm{sec}$ without load [16]. China has the fastest maglev which runs at $431 \mathrm{kph}$ and Japan has tested one that runs at $581 \mathrm{kph}$. For optimal safety the passengers and crew are insulated from the magnetic fluxes at least by magnetic fabric insulators.

Ordinary bearings to minimize friction between the train and the track (Figure 7) are impractical as they wear out quickly due to the great speed of the train.

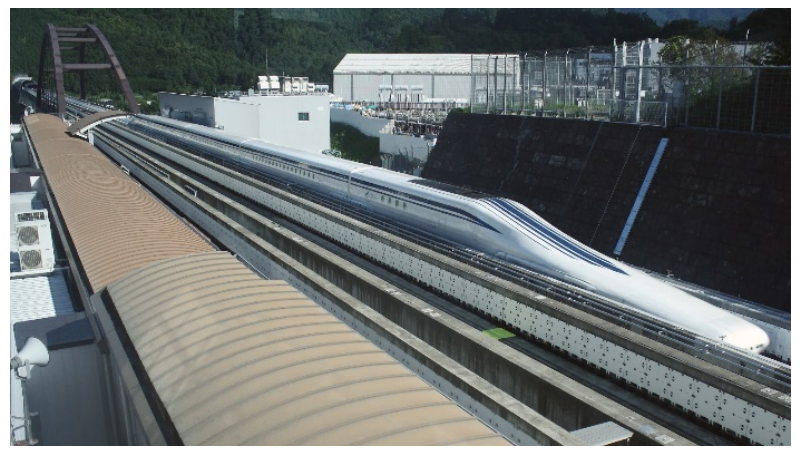

Figure 8. A 5-car long nosed maglev (for least aerodynamic resistance). The magnetic engine is in the lead car.

Instead, fat bar magnets line the inner surfaces of the full length of the latch on each side of the train's car. Opposite them, an array of magnets of the same poles is placed on both edges of the track (Figure 10). 


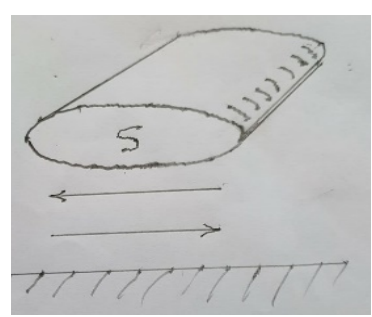

Figure 9. The maglev engine is an elliptical bar magnet. Its lateral axis is parallel to the track when the train is at rest. The first arrow points in the direction of the magnetic flux of the maglev's engine and the second arrow points in the direction of the magnetic fluxes of the magnets on the track (not shown). Since the two fluxes are opposite they repel each other.

They are spaced 1/3 the length of each car and line the full stretch of the track so that for each car there is at least one pair of repulsive magnets near its ends.

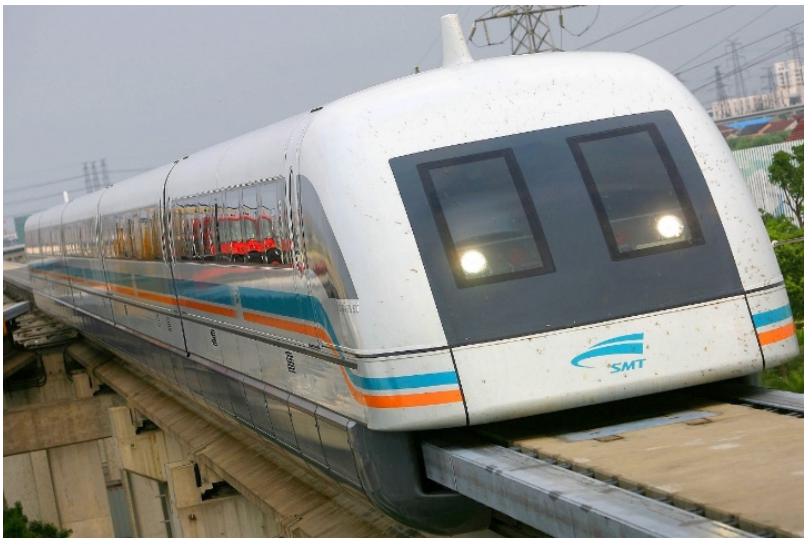

Figure 10. The maglev showing the track as the guide and holder at the same time.

The magnets serve as frictionless bearings, springs, stabilizers and shock absorbers all at once and provide the passenger fast, smooth, quiet and comfortable ride. The magnetic flux of a magnet does not erode unless it is dropped into a hard surface. This does not happen to the magnets in the train and the track. There is no wear and tear in the maglev and the track because there is no moving part and no friction. Therefore, maintenance and operational costs equal zero since the train floats on the magnetic fluxes without friction. Only the force of inertia in starting and stopping needs to be overcome by the magnetic engine and the break mechanism. Corrosion is mainly superficial and does not affect the internal structure of the magnet. But still, it is a problem in the long haul. The remedy is non-ferromagnetic anticorrosive coating for the magnets. Putting the magnets next to each other on the track is impossible due to Coloumb's law. Therefore, magnetic insulators must be placed between them. This avoids, at the same time, magnetic deterioration due to magnetic interpenetration.

\section{Break Mechanism for Maglev}

At one $\mathrm{km}$ from the train station, cut the length of each track magnet by $6.25^{\prime \prime}$ at each end (see Figure 10). Insert an array of elliptical magnets 6" thick, of opposite polarity as the track magnets, on both ends of the track magnets from one $\mathrm{km}$ away from the station to the far end of the platform. We call them track brake pads. Insert magnetic insulator between the track magnets and the track brake pads. On the train and in front of the magnetic engine, place a pair of elliptical magnets aligned with the ends of the magnetic engine. We call these pair of magnets train brake pads. When the train is running, the train brake pads' lateral axes are horizontal. At $1.5 \mathrm{~km}$ from the station the magnetic engine's lateral axis is pulled up to horizontal position. The train will continue to run at the same speed due to inertia. When the pair of train brake pads hits the one-km mark from the station, the brake pads' back sides are tilted downward gradually to pull back the train to a stop at the train station. 
Being powered by the free, clean and indestructible dark matter - magnetic fluxes - the maglev is the ultimate in technology. But there is a drawback: the cost of construction is prohibitive: $\$ 50$ billion for a 150-km track. The technology proposed here will recover the cost quickly and generate electricity huge enough to do away with fossil, nuclear and geothermal fuel and still export electricity worldwide.

\section{ELECTRIC POWER GENERATOR RUN BY DARK MATTER}

Mount a system of parallel vertical conductors on the roof of any train along the full length of the train (Figure 11). They should be close together as much as possible but safely apart from each other to accommodate huge number of conductors covering the entire roof. They may be grouped into blocks or groups to generate desired voltage.

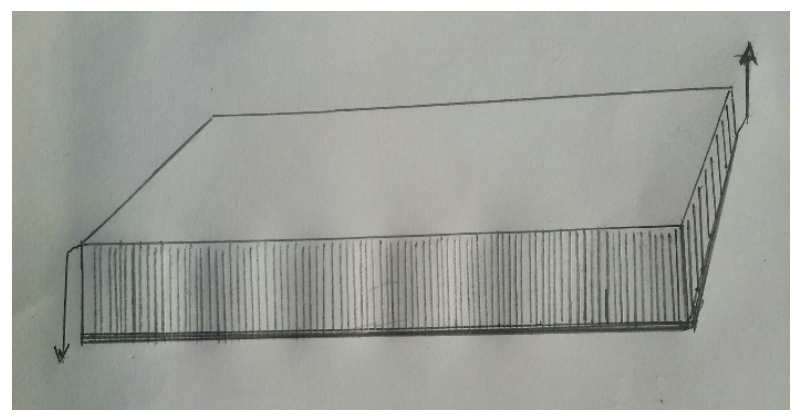

Figure 11. Parallel conductors are mounted on the roof of a train; they cut magnetic lines of force (magnetic flux) provided by an array of C-magnets as the train moves along the track and generate electricity.

Install an array of C-magnets of the same polarity over the track through the entire stretch of the track at the right height above the track so that the conductors of Figure 11 cut across their magnetic lines of force as the train passes through to generate huge electric power for the power grid. Magnetic insulators should be inserted between the C-magnets to break magnetic flux coherence and counter the repulsion between the magnets due to Coulomb's law and neutralize the interpenetration between them that tends to degrade their strength. The outlet is connected to a reverse rectifier to convert DC to AC before connecting to the power grid.

If we install such power generators on all track systems, maglev or conventional, country and city rails, huge electric power can be generated for local use and still accumulate surplus that can be exported worldwide through power cables.

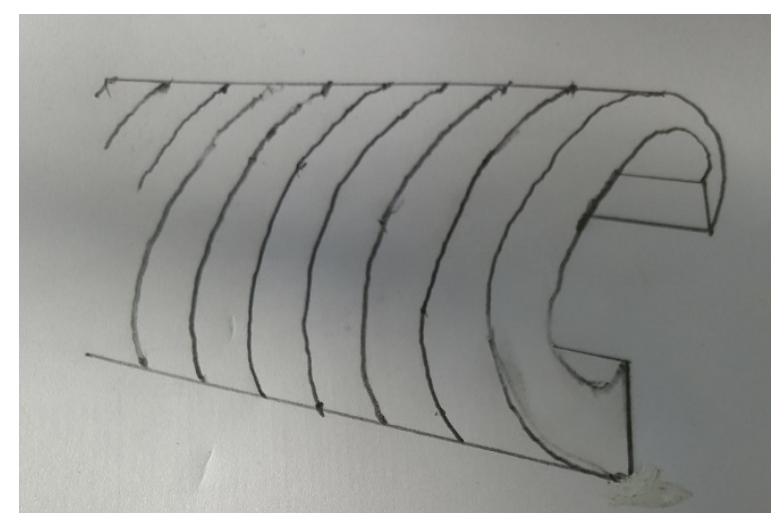

Figure 12. An array of C-magnets mounted above the railroad track along its full stretch at a height so that, as the train passes through, the parallel conductors on the train's roof (Figure 8) cut across the magnetic lines of force provided by the $\mathrm{C}$-magnets and generate $\mathrm{DC}$ current. It is converted to $\mathrm{AC}$ by a reverse rectifier and connected to the power grid. 
Then we can do away with power plants run by the dirty fossil, nuclear and geothermal fuel. This way we can liberate planet Earth from the impending environmental disaster it now faces. Moreover, left alone, nature heals itself and reverses the negative legacy of fossil-, nuclear- and geothermal-powered technologies within limits. Furthermore, this is the only way for the Third World to liberate themselves from underdevelopment faster than the developed countries and the NIC did.

\section{MAGNETIC CHARGER RUN BY DARK MATTER}

A modification of the maglev can be devised at smaller scale for motor vehicles. Mount parallel conductors on the roof of a car (or under the chassis). The magnetic charger consisting of an array of C-magnets can be constructed along a highway by-pass so that the parallel conductors cut across their magnetic lines of force as the car passes through. The direct current generated is stored in a parallel tandem of lithium batteries to power the car for the next hundred kilometers or so depending on the number of magnets in the array. As in conventional motor vehicles a charger may be installed every $30 \mathrm{~km}$. Chargers for trucks and busses may be installed separately.

Alternatively, the magnetic charger can be replaced by electromagnetic charger run by the power grid whichever costs less.

Super magnets can power cargo ships and ocean liners. The author has seen a small prototype of six 2-inch diameter disc magnets that yields 720 watts of electricity. If so many of these prototypes are connected parallel to each other tens of thousand megawatts of power can be generated. The author has also a sketch of a potential magnetic engine for space vehicle (e.g., UFO) using the Searle effect.

\section{NOTE}

Magnets last forever unless they are dropped or pounded vigorously neither of which occurs in these cases. Corrosion is superficial and does not affect the internal arrangement of the atom. Even corrosion can be minimized with effective non-ferromagnetic coating.

\section{References}

1. E. E. Escultura, The Solution of the Gravitational n-Body Problem, Nonlinear Analysis, Series A: Theory, Methods and Applications, 30(8), Dec. 1997, 5021 - 5032.

2. L. C. Young, Mathematicians and Their Times, North-Holland, 1980, Amsterdam.

3. P. J. Davies and R. Hersch, The Mathematical Experience, Birkhäuser, Boston, 1981.

4. E. E. Escultura, The Trajectories, Reachable Set, Minimal Levels and Chains of Trajectories of a Control System, Ph.D. Thesis, University of Wisconsin, 1970.

5. E. E. Escultura, Exact Solutions of Fermat's Equation (Definitive resolution of Fermat's Last Theorem, Nonlinear Studies), 5(2), 1998, 227 - 254.

6. E. E. Escultura, Rational Thought, Cognition and Knowledge International Education Research, 3(1), 2015, 21 - 37; http://www.todayscience.org/IER/article/ier.v3i1p21.pdf.

7. E. E. Escultura, The Resolution of the Great 20th Century Debate in the Foundations of Mathematics, 6(2015), 144-158; http://file.scirp.org/Html/3-5301056_63915.htm.

8. E. E. Escultura, Quantum Gravity, SciFed Journal of Quantum Physics, 2(1), 2017, 1 - 16; http://scifedpublishers.com/fulltext/quantum-gravity/21958.

9. Newton's Three Laws of Motion; http://teachertech.rice.edu/Participants/louviere/Newton/.

10. Laws of thermodynamics; https://en.wikipedia.org/wiki/Laws_of_thermodynamics.

11. E. E. Escultura, The Pillars of the New Physics and Some Updates, Nonlinear Studies, 14(3), 2007, 241 260. 
12. E. E. Escultura, The grand unified theory, Nonlinear Analysis: A-Series: Theory, Methods and Applications, 69(3), 2008, $823-831$.

13. Science, Starbirth, Gamma Blast Hint at Active Early Universe, 282(5395), December, 1998, p. 806.

14. Science, Glow Reveals Early Star Nurseries, 281(5375), July 1998, 332 - 333.

15. E. E. Escultura, The Logic and Fundamental Concepts of the Grand Unified Theory, Journal of Modern Physics, (SCIRP), 4(8A), 2013, 213 - 222; http://file.scirp.org/Html/21-7501396_36280.htm.

16. V.A. Atsukovsky, General Ether-Dynamics, Simulation of the Matter Structures and Fields on the Basis of the Ideas About the Gas-like Ether, Energoatomizdat, Moscow, 1990.

17. E. E. Escultura, Extended Geometrical and Generalized Fractals, Chaos and Applications, in Kyle J. Brennan, ed, Handbook on the Classification and Application of Fractals, Nova Publishers, 2011, 1 - 39; http://www.scirp.org/reference/ReferencesPapers.aspx?ReferenceID=1848390.

18. E. E. Escultura, The Constructivist Real Number System, Advances in Pure Mathematics, 6(9), 2016,597 607; http://www.scirp.org/journal/apm/.

19. E. E. Escultura, Diophantus: Introduction to Mathematical Philosophy (With Probabilistic Solution of Fermat and Other Applications), Kalikasan Press, 1993, Manila.

I. L. Gerlovin, The Foundations of United Theory of Interactions in a Substance, Leningrad: Energoattomizdat, 1990.

20. Electron Spin; http://hyperphysics.phy-astr.gsu.edu/hbase/spin.html.

21. E. E. Escultura, Turbulence: Theory, Verification and Applications, Nonlinear Analysis, Series A: Theory, Methods and Applications, 47(2001), 5955 - 5966.

22. E. E. Escultura, Chaos, Turbulence and Fractal: Theory and Applications, International J. Modern Nonlinear Theory and Applications, 2(3), 2012, 176 - 185; http://www.scirp.org/journal/paperInformPaperInformation.aspx?Paper ID=368 49.

23. H. A. Nieper, Revolution in Technology, Medicine and Society, Management Interessengemeinschaft für Tachyon-Feld-Energie, 1983, Odenburg, FRG.

24. CERN; https://en.wikipedia.org/wiki/CERN.

25. E. E. Escultura, Qualitative Model of the Atom, its Components and Origin in the Early Universe, Nonlinear Analysis: Real World Applications, 11(2009), 29 - 38.

26. Science, Neutrinos weigh in, 282(5397), 1998, $2158-2159$.

27. Search for neutrino mass is a big stretch for three labs, 283(5405), 1999, 928 - 929.

28. E. E. Escultura, The Mathematics of the Grand Unified Theory, Nonlinear Analysis, A-Series: Theory, Methods, 71(2009), e420 - e431.

29. E. Merchbacher, Quantum Mechanics, 2nd ed., John Wiley \& Sons, Inc. 1970, New York.

30. E. E. Escultura, Qualitative Mathematics and Modeling: Theoretical and Practical Applications, LAP LAMBERT Academic Publishing, Saarbrücken, 2013, https://www.amazon.com/Qualitative-MathematicsModeling-Theoretical-Applications/dp/3659305847.

31. D. Pendick, Fires in the Sky, Earth, 20, June 1996, $62-64$.

32. E. E. Escultura, Is Human Activity Linked to Climate Change? Atmospheric and Climate Sciences, 4(2014), 305 - 316; http://file.scirp.org/Html/15-4700251_45173.htm.

33. Donald M. Ginsberg, Superconductivity; https://www.britannica.com/science/superconductivity. 